\title{
THE ABSOLUTE MAGNITUDE OF RR LYRAE STARS DERIVED FROM THE HIPPARCOS CATALOGUE
}

\author{
Takuji Tsujimoto ${ }^{1}$, Masanori Miyamoto ${ }^{1}$, Yuzuru Yoshii ${ }^{2,3}$ \\ ${ }^{1}$ National Astronomical Observatory, Mitaka, Tokyo 181, Japan \\ ${ }^{2}$ Institute of Astronomy, Faculty of Science, University of Tokyo, Mitaka, Tokyo 181, Japan \\ ${ }^{3}$ Research Center for the Early Universe, School of Science, University of Tokyo, Bunkyo-ku, Tokyo 113, Japan
}

\begin{abstract}
The present determination of the absolute magnitude $M_{V}(\mathrm{RR})$ of RR Lyrae stars is twofold, relying upon Hipparcos proper motions and trigonometric parallaxes separately. First, applying the statistical parallax method to the proper motions, we find $<M_{V}(\mathrm{RR})>=0.69 \pm 0.10$ for 99 halo RR Lyraes with $<[\mathrm{Fe} / \mathrm{H}]>=-1.58$. Second, applying the Lutz-Kelker correction to the RR Lyrae HIP95497 with the most accurately measured parallax, we obtain $M_{V}(\mathrm{RR})=(0.58-0.68)_{-0.31}^{+0.28}$ at $[\mathrm{Fe} / \mathrm{H}]=-1.6$. Furthermore, allowing full use of low accuracy and negative parallaxes as well for $125 \mathrm{RR}$ Lyraes with $-2.49 \leq[\mathrm{Fe} / \mathrm{H}] \leq 0.07$, the maximum likelihood estimation yields the relation, $M_{V}(\mathrm{RR})=(0.59 \pm 0.37)+(0.20 \pm 0.63)([\mathrm{Fe} / \mathrm{H}]+1.60)$, which formally agrees with the recent preferred relation. The same estimation yields again $\left\langle M_{V}(\mathrm{RR})\right\rangle=0.65 \pm 0.33$ for the 99 halo RR Lyraes. Although the formal errors in the latter three parallax estimates are rather large, all of the four results suggest the fainter absolute magnitude, $M_{V}(\mathrm{RR}) \approx 0.6-0.7$ at $[\mathrm{Fe} / \mathrm{H}]=-1.6$. The present results still provide the lower limit on the age of the universe which is inconsistent with a flat, matter-dominated universe and current estimates of the Hubble constant.
\end{abstract}

Subject headings: astrometry — stars: luminosities — stars: kinematics — stars: statistics 


\section{INTRODUCTION}

The absolute magnitude of RR Lyrae stars $M_{V}(\mathrm{RR})$ is an indicator of distance and age in the Galaxy. In particular it is a key parameter for determining the age of Galactic globular clusters (GCs) which should place a lower limit on the age of the universe. However, the values of $M_{V}(\mathrm{RR})$ reported so far defies a consensus and splits into faint and bright values.

The faint value of $M_{V}(\mathrm{RR})=0.70-0.75$ at the characteristic halo metallicity $[\mathrm{Fe} / \mathrm{H}]=-1.6$, which gives a shorter distance scale or larger age of clusters, is derived from halo RR Lyraes in the fields by the statistical parallax method (Hawley et al. 1986; Barnes \& Hawley 1986; Strugnell, Reid, \& Murray 1986; Layden et al. 1996) and by the Baade-Wesselink analysis (Liu \& Janes 1990a; Jones et al. 1992). On the other hand, the bright value of $M_{V}(\mathrm{RR})=0.44$ at $[\mathrm{Fe} / \mathrm{H}]=-$ 1.9, which gives a longer distance scale or smaller age, is derived from RR Lyraes in the GCs of the LMC, assuming a distance modulus to the LMC to be 18.5 (Walker 1992). Sandage's pulsation theory (Sandage 1993) and RR Lyraes in the metal-poor GCs of M5 and M92 (Storm, Carney, \& Latham 1994) also support the bright value, but this is not the case for RR Lyares in M4 (Liu \& Janes 1990b).

Such a dichotomy in current estimates of $M_{V}(\mathrm{RR})$ is the dominant source of uncertainty in the age determination of GCs. The faint $M_{V}(\mathrm{RR})$ raises a wellknown problem such that the age of GCs exceeds the age of the universe if the Hubble constant is adopted as $H_{0}=60-80 \mathrm{~km} \mathrm{~s}^{-1} \mathrm{Mpc}^{-1}$, and this is the central argument in favor of nonzero cosmological constant (Bolte \& Hogan 1995). However, a possibility of swaying back to the bright $M_{V}(\mathrm{RR})$ has recently been discussed since the preference of its bright value was inferred from the Hipparcos measurements of parallaxes of Cepheids (Feast \& Catchpole 1997) and subdwarfs (Reid 1997).

The astrometric observations of field RR Lyraes have been available to us as a part of the Hipparcos programme assigned to the proposers in 1982. This paper is therefore the first report in the main journal on the direct determination of $M_{V}(\mathrm{RR})$ from statistical treatments of Hipparcos proper motions and trigonometric parallaxes of RR Lyraes (cf., Tsujimoto, Miyamoto, \& Yoshii 1997; Fernley et al. 1997). The derived results from the proper motions in $\S 2$ and from the parallaxes in $\S 3$ have confirmed the faint $M_{V}(\mathrm{RR})$, thus suggesting that the so-called age dis- crepancy would not be resolved or alleviated yet.

\section{2. $M_{V}(\mathrm{RR})$ DERIVED FROM HIPPARCOS PROPER MOTIONS}

The Hipparcos proper motion system is inertial, on a global sense, within the error $\pm 0.25 \mathrm{mas} / \mathrm{yr}$ and the mean error $\left(\sigma_{\mu}\right)_{\mathrm{H}}$ of the Hipparcos proper motions $\left[\left(\mu_{\alpha}^{*}\right)_{\mathrm{H}},\left(\mu_{\delta}\right)_{\mathrm{H}}\right]$ for the RR Lyraes considered here is [ \pm 3 mas/yr, \pm 2 mas/yr]. Thus, the previous proper motions are remarkably improved by the Hipparcos mission. The most essential part of improvements of the previous proper motions $\left[\left(\mu_{\alpha}^{*}\right)_{\mathrm{p}},\left(\mu_{\delta}\right)_{\mathrm{p}}\right]$ may be described by the rotation vector $\boldsymbol{\Omega}$ of the previous proper motion system with respect to the Hipparcos system. The vector components $\left(\Omega_{x}, \Omega_{y}, \Omega_{z}\right)$ in the equatorial rectangular coordinates are derived from the equations of condition:

$$
\left.\begin{array}{rl}
{\left[\left(\mu_{\alpha}^{*}\right)_{\mathrm{p}}-\left(\mu_{\alpha}^{*}\right)_{\mathrm{H}}\right]=} & -\Omega_{x} \sin \delta \cos \alpha-\Omega_{y} \sin \delta \sin \alpha \\
{\left[\left(\mu_{\delta}\right)_{\mathrm{p}}-\left(\mu_{\delta}\right)_{\mathrm{H}}\right]=} & +\Omega_{x} \sin \alpha \quad-\Omega_{y} \cos \alpha \\
& +\Omega_{z} \cos \delta
\end{array}\right\} .
$$

Applying eq.[1] to the proper motions of 99 halo RR Lyraes with $<[\mathrm{Fe} / \mathrm{H}]>=-1.58$, which are common to Table 2 compiled by Layden et al. (1996) and Hipparcos Catalogue, we have

$$
\left.\begin{array}{l}
\Omega_{x}=+1.61 \pm 0.15 \mathrm{mas} / \mathrm{yr} \\
\Omega_{y}=+4.31 \pm 0.13 \mathrm{mas} / \mathrm{yr} \\
\Omega_{z}=-1.27 \pm 0.13 \mathrm{mas} / \mathrm{yr}
\end{array}\right\} .
$$

Thus, the previous proper motions are systematically improved by the amount of $|\boldsymbol{\Omega}| \sim 5 \mathrm{mas} / \mathrm{yr}$ larger than the mean error $\left(\sigma_{\mu}\right)_{\mathrm{H}}$ in Hippracos proper motions for the RR Lyraes. Encouraged by the clear improvement indicated by eq.[2], we apply the statistical parallax method to Hipparcos proper motions to improve $M_{V}(\mathrm{RR})$ previously determined.

The method is a direct application of well-understood kinematics of sample stars to the observations (proper motions, radial velocities, and apparent magnitudes), when a single luminosity class of sample stars can be specified within a small intrinsic magnitude dispersion. The present maximum likelihood statistical analysis of RR Lyraes is similar to the one done on RR Lyraes by Hawley et al. (1986). Introducing a model that predicts the spatial motion of each star at each position in the Galaxy, we estimate the model parameters by the maximum likelihood method. The 
Table 1: Results of statistical parallax analysis

\begin{tabular}{cccccccccc}
\hline & $N_{\text {stars }}$ & $\langle[\mathrm{Fe} / \mathrm{H}]>$ & $U_{\odot}$ & $V_{\odot}$ & $W_{\odot}$ & $\sigma_{U}$ & $\sigma_{V}$ & $\sigma_{W}$ & $M_{V}(\mathrm{RR})$ \\
\hline Hawley et al. (1986) & 77 & - & $+21 \pm 19$ & $-184 \pm 17$ & $-4 \pm 11$ & $166 \pm 76$ & $114 \pm 52$ & $91 \pm 40$ & $0.73 \pm 0.18$ \\
Layden et al. (1996) & 162 & -1.61 & $-9 \pm 14$ & $-210 \pm 12$ & $-12 \pm 8$ & $168 \pm 13$ & $102 \pm 8$ & $97 \pm 7$ & $0.71 \pm 0.12$ \\
Fernley et al. (1997) & 69 & -1.66 & - & - & - & - & - & - & $0.73 \pm 0.18$ \\
this work & 99 & -1.58 & $-12 \pm 17$ & $-200 \pm 11$ & $+2 \pm 3$ & $161 \pm 13$ & $105 \pm 9$ & $88 \pm 7$ & $0.69 \pm 0.10$ \\
\hline
\end{tabular}

present model includes a reflex solar motion with respect to the galactic center and an ellipsoidal velocity distribution for RR Lyraes, but neglects the differential galactic rotation. Assuming that the velocity residuals $\boldsymbol{\nu}$, the observed velocity minus the velocity expected from the model, follow a Gaussian distribution with zero mean, we have the logarithmic likelihood $\ln L$ of the aggregate of all the residuals:

$$
\ln L=-\frac{1}{2} \Sigma \ln \left(|\boldsymbol{M}|+\boldsymbol{\nu}^{\prime} \boldsymbol{M}^{-1} \boldsymbol{\nu}\right)+\text { constant , }
$$

where $\boldsymbol{M}$ is the covariance tensor given by the expectation $\boldsymbol{M}=\left\langle\boldsymbol{\nu} \boldsymbol{\nu}^{\prime}\right\rangle$.

The model parameters describe the velocity components $\left(U_{\odot}, V_{\odot}, W_{\odot}\right)$ of the reflex solar motion with $U, V$, and $W$ axes pointing to the galactic center, the galactic rotation, and the north galactic pole, respectively, the velocity dispersion components $\left(\sigma_{U}, \sigma_{V}, \sigma_{W}\right)$, the covariances $\left(\sigma_{U V}, \sigma_{U W}, \sigma_{V W}\right)$, the absolute magnitude $M_{V}(\mathrm{RR})$, and the intrinsic magnitude dispersion $\sigma_{M}$ in $M_{V}(\mathrm{RR})$. The observational errors $\sigma_{V_{r}}$ in the radial velocity and $\left(\sigma_{\mu_{\alpha}}, \sigma_{\mu_{\delta}}\right)$ in the proper motions for each star are taken from Layden (1996) and Hipparcos Catalogue, respectively. The intrinsic dispersion $\sigma_{M}$ is parameterized by $\sigma_{k}$, the dispersion in the distance scale parameter $k$. Here we assume $\sigma_{k}=0.1$ (Layden et al. 1996). The present model therefore incorporates 10 parameters, which are solved by the numerical minimization technique of simplex optimization.

Combining Hipparcos proper motions with the data of $[\mathrm{Fe} / \mathrm{H}]$, radial velocity, apparent magnitude, and interstellar extinction taken from Layden et al. (1996) and Layden (1994), we have carried out the present analysis for 99 halo RR Lyraes with $<[\mathrm{Fe} / \mathrm{H}]>=-1.58$, and found that all of the covariances $\left(\sigma_{U V}, \sigma_{U W}, \sigma_{V W}\right)$ are nearly equal to zero. Therefore, we have repeated the similar analysis to determine 7 parame- ters, putting all of the covariances equal to zero. The results are shown in Table 1 . We find $\left\langle M_{V}(\mathrm{RR})\right\rangle$ $=0.69 \pm 0.10$ together with the solar motion with respect to the galactic center $\left(U_{\odot}, V_{\odot}, W_{\odot}\right)=(-12 \pm 17$,$200 \pm 11,+2 \pm 3) \mathrm{km} \mathrm{s}^{-1}$ and the velocity dispersions $\left(\sigma_{U}, \sigma_{V}, \sigma_{W}\right)=(161 \pm 13,105 \pm 9,88 \pm 7) \mathrm{km} \mathrm{s}^{-1}$. The RR Lyrae stars as a whole rotate with $V_{\text {rot }} \approx 30 \mathrm{~km}$ $\mathrm{s}^{-1}$, assuming the local standard of rest $V_{\mathrm{LSR}}=220$ $\mathrm{km} \mathrm{s}^{-1}$ and the solar motion of $16.5 \mathrm{~km} \mathrm{~s}^{-1}$ in the direction $l=53^{\circ}$ and $b=25^{\circ}$. These results are in good agreement with those recently obtained by Hawley et al. (1986), Layden et al. (1996), and Fernley et al. (1997) (see Table 1) within the error estimates. Thus, the absolute magnitude of RR Lyraes derived from Hipparcos proper motions, $\left\langle M_{V}\right\rangle \approx 0.70$ at $<[\mathrm{Fe} / \mathrm{H}]>\approx-1.6$, confirms the recent results from the ground-based observations.

In general, a linear relation between $M_{V}(\mathrm{RR})$ and $[\mathrm{Fe} / \mathrm{H}]$ has been assumed. The slope of $M_{V}(\mathrm{RR})$ $[\mathrm{Fe} / \mathrm{H}]$ relation has a strong influence on the inferred age differences among the globular clusters, which give a measure of the dynamical timescale for halo formation. The Baade-Wesselink results give the values of $M_{V}(\mathrm{RR})$ over the range of $[\mathrm{Fe} / \mathrm{H}]=-2.2$ to 0 , which are fitted by the relation: $M_{V}(\mathrm{RR})=$ $(1.02 \pm 0.03)+(0.16 \pm 0.03)[\mathrm{Fe} / \mathrm{H}]($ Jones et al. 1992) or $M_{V}(\mathrm{RR})=(1.04 \pm 0.10)+(0.21 \pm 0.05)[\mathrm{Fe} / \mathrm{H}]$ (Skillen et al. 1992). However, the controversial situation holds also for determining the slope of $M_{V}(\mathrm{RR})-[\mathrm{Fe} / \mathrm{H}]$ relation. Sandage's pulsation theory gives much steeper slope, whereas statistical parallax analyses (Hawley et al. 1986; Strugnell et al. 1986; Layden et al. 1996) support very weak (or no) dependence of $M_{V}(\mathrm{RR})$ on $[\mathrm{Fe} / \mathrm{H}]$. It is, of course, possible to incorporate the linear relation between $M_{V}(\mathrm{RR})$ and $[\mathrm{Fe} / \mathrm{H}]$ into the present analysis by dividing the available sample into bins of $[\mathrm{Fe} / \mathrm{H}]$, but the available number (26) of Hipparcos RR Lyraes with $[\mathrm{Fe} / \mathrm{H}]>-1.0$ is too small to yield the reliable relation over the wide range of 
$[\mathrm{Fe} / \mathrm{H}]$.

\section{3. $M_{V}(\mathrm{RR})$ DERIVED FROM HIPPARCOS PARALLAXES}

Fig. 1. - The comparison between Hipparcos parallaxes $\pi_{\mathrm{H}}$ of $125 \mathrm{RR}$ Lyraes and the photometric parallaxes $\pi_{\mathrm{p}}$ (see eq.[5]). The straight line indicates the relation $\pi_{\mathrm{H}}=\pi_{\mathrm{p}}$.

The relative error $\sigma_{\pi} / \pi_{\mathrm{H}}$ for 173 Hipparcos RR Lyraes with the standard error $\sigma_{\pi}$ of Hipparcos parallaxes $\pi_{\mathrm{H}}$ distributes such that each number of stars with $\sigma_{\pi} / \pi_{\mathrm{H}}$ less than 1 and larger than 1 is 51 and 65 , respectively. The remaining 57 stars have even negative parallaxes. Thus, almost all of the parallaxes are measured with very large error, except one case (HIP95497 $=\mathrm{RR}$ Lyr) with $\sigma_{\pi} / \pi_{\mathrm{H}}=0.135$. In Figure 1 Hipparcos parallaxes $\pi_{\mathrm{H}}$ are compared with the photometric ones $\pi_{\mathrm{p}}$ defined by $r_{\mathrm{p}}(\mathrm{kpc})=$ $10^{0.2\left(V-M_{V}(\mathrm{RR})-10\right)}$, where $V$ is the apparent magnitude corrected for interstellar extinction and $M_{V}(\mathrm{RR})$ the calibrated absolute magnitude given by the relation

$$
M_{V}(\mathrm{RR})=a+b([\mathrm{Fe} / \mathrm{H}]-c)
$$

with $a=0.91, b=0.20, c=-1.60$ (see eq.[5]). The filled and open circles in the figure indicate the parallaxes with $\sigma_{\pi} / \pi_{\mathrm{H}}$ smaller than 1 and others, respectively. It is noticed that the individual deviation of Hipparcos parallaxes from the photometric ones is extremely large, but Hipparcos parallaxes including low quality and negative ones as a whole agree with the photometric ones.

The statistical properties of Hipparcos parallaxes of their errors have been investigated extensively by Arenou et al. (1995), who have shown that Hipparcos parallaxes are free from any global zero-point error up to \pm 0.1 mas and their errors are normally distributed with zero mean. This means that Hipparcos parallaxes including even the negative ones for any ensemble of stars are statistically well-behaved. This is the case for the ensemble of RR Lyraes as well, as is shown in Figure 1. These statistical regularities of Hipparcos parallaxes suggest a relevant technique for estimating statistically the absolute magnitude $M_{V}(\mathrm{RR})$ of RR Lyraes to be applicable to the ensemble of $R R$ Lyrae parallaxes, even though each individual parallax measured is largely erroneous, giving negative one as well. Here we attempt to derive $M_{V}(\mathrm{RR})$ directly from Hipparcos parallaxes, relying upon two statistical methods to find the unbiased estimate.

a) Lutz-Kelker Correction

Lutz \& Kelker (1973) have shown that only when the relative error $\sigma_{\pi} / \pi^{\prime}$ is smaller than 0.175 , an unbiased estimate of the absolute magnitude can be retrieved from the measured parallaxes $\pi^{\prime}$. We have only one case of HIP95497 with $\sigma_{\pi} / \pi_{\mathrm{H}}=0.135$, to which Lutz-Kelker correction is applicable. The uncorrected absolute magnitude of this star is $M_{V}(\mathrm{RR})$ $=0.82_{-0.31}^{+0.28}$, which is derived formally from the measured parallax. On the basis of Hanson's (1979) formulation to estimate the correction, we have the calibrated absolute magnitude range $M_{V}(\mathrm{RR})=(0.62-$ $0.72)_{-0.31}^{+0.28}$ for HIP95497 $([\mathrm{Fe} / \mathrm{H}]=-1.37)$, corresponding to the assumed spatial density of RR Lyraes $n(r)=$ const. and $n(r) \propto r^{-2}$ as the lower and upper bounds, respectively. If we assume $b=0.20$ in eq. [4], the above range of $M_{V}(\mathrm{RR})$ for HIP95497 corresponds to $M_{V}(\mathrm{RR})=(0.58-0.68)_{-0.31}^{+0.28}$ at $[\mathrm{Fe} / \mathrm{H}]=-$ 1.6 .

b) Maximum Likelihood Calibration

In order to retrieve statistically an unbiased estimate of the absolute magnitude together with its intrinsic dispersion for a single luminosity class of magnitude limited stars from their measured parallaxes with errors, Smith $(1987,88)$ has proposed a maximum likelihood principle for correcting simultaneously the Malmquist bias and the Lutz-Kelker bias, allowing full use of low accuracy and negative paral- 
laxes.

In line with the above principle we attempt to find the best esimate of the absolute magnitude $M_{V}(\mathrm{RR})$ and of the intrinsic magnitude dispersion $\sigma_{M}$ for 125 Hipparcos RR Lyraes in the metallicity range $2.49 \leq[\mathrm{Fe} / \mathrm{H}] \leq 0.07$. We use here the numerical algorithm developed by Ratunatunga \& Casertano (1991). Maximizing the likelihood function given by the product of the probabilities $p\left(\pi_{\mathrm{H}}\right)$ of all the RR Lyraes considered, we obtain the best estimate of $\sigma_{M}, a$ and $b$ in eq. [4], where we adopt $c=-1.60$, taking into account the fact that the present distribution of $\mathrm{RR}$ Lyraes shows a peak at $[\mathrm{Fe} / \mathrm{H}] \approx-1.6$. Setting the allowable range of the measured parallaxes as $\left[\pi_{\text {lower }}, \pi_{\text {upper }}\right]=[-\infty,+\infty]$, and assuming the number density of RR Lyraes in space to be constant, we find the $M_{V}(\mathrm{RR})-[\mathrm{Fe} / \mathrm{H}]$ relation:

$M_{V}(\mathrm{RR})=(0.59 \pm 0.37)+(0.20 \pm 0.63)([\mathrm{Fe} / \mathrm{H}]+1.60)$

with $\sigma_{M}=2.6 \times 10^{-4} \pm 0.29$. It is noticed that although the errors in the parameters estimated are large, the present $M_{V}(\mathrm{RR})-[\mathrm{Fe} / \mathrm{H}]$ relation shows a formal agreement with the preferred relation $M_{V}(\mathrm{RR})=$ $0.98+0.20[\mathrm{Fe} / \mathrm{H}]$ proposed by Chaboyer, Demarque, \& Sarajedini (1996) without quoting error estimates. The insensitivity of the relation given by eq. [5] has been checked by putting $\sigma_{M}=0.1-0.2$ (Layden et al. 1996), $\left[\pi_{\text {lower }}, \pi_{\text {upper }}\right]=[0,+\infty]$, and $n(r) \propto r^{-2}$ (Reid 1997), respectively.

The large standard errors in eq. [5] are owing to the small numbers of degrees of freedom in the present statistics. To increase the freedom, putting $b=0$ in eq.[4], we apply the same method to the 99 halo RR Lyraes with $<[\mathrm{Fe} / \mathrm{H}]>=-1.58$, whose absolute magnitude has been determined already on the basis of the statistical parallax method. Then, we find $<M_{V}(\mathrm{RR})>=0.65 \pm 0.33$ and $\sigma_{M}=4.1 \times 10^{-6} \pm 0.39$ at $\langle[\mathrm{Fe} / \mathrm{H}]\rangle=-1.58$. This absolute magnitude can be compared with $M_{V}(\mathrm{RR})=0.59 \pm 0.37$ at $[\mathrm{Fe} / \mathrm{H}]=-1.6$ given by eq.[5]. The validity of the present statistical analysis has been further checked by inspecting the distribution $\left(\pi_{\mathrm{H}}-\pi_{\mathrm{p}}\right) / \sigma_{\pi}$ for the $125 \mathrm{RR}$ Lyrae stars. The median of the distribution is shifted only by 0.08 from the Gaussian.

\section{CONCLUSION}

The present derivation of the absolute magnitude of RR Lyrae stars is twofold, relying upon Hipparcos proper motions and trigonometric parallaxes. First, applying the statistical parallax method to Hipparcos proper motions of 99 halo RR Lyraes, we have found

$$
<M_{V}(\mathrm{RR})>=0.69 \pm 0.10 \text { at }<[\mathrm{Fe} / \mathrm{H}]>=-1.58 \text {. }
$$

Second, applying the Lutz-Kelker correction to the most accurate parallax of HIP95497 (RR Lyr) with $\sigma_{\pi} / \pi_{\mathrm{H}}=0.135$ and $[\mathrm{Fe} / \mathrm{H}]=-1.37$, we have the magnitude range $M_{V}(\mathrm{RR})=(0.62-0.72)_{-0.31}^{+0.28}$, which yields

$$
M_{V}(\mathrm{RR})=(0.58-0.68)_{-0.31}^{+0.28} \text { at }[\mathrm{Fe} / \mathrm{H}]=-1.6 \text {, }
$$

given the slope 0.20 of the $M_{V}(\mathrm{RR})-[\mathrm{Fe} / \mathrm{H}]$ relation. The lower and upper bounds in eq.[7] correspond to the assumed spatial density of RR Lyraes $n(r)=$ const. and $n(r) \propto r^{-2}$, respectively. Furthermore, applyng the maximum likelihood algorithm to Hipparcos parallaxes including low accuracy and negative ones as well for $125 \mathrm{RR}$ Lyraes with $2.49 \leq[\mathrm{Fe} / \mathrm{H}] \leq 0.07$, we have found the relation (5) which gives

$$
M_{V}(\mathrm{RR})=0.59 \pm 0.37 \text { at }[\mathrm{Fe} / \mathrm{H}]=-1.6 \quad .
$$

Applying the same method to the 99 halo RR Lyraes, we have found

$$
<M_{V}(\mathrm{RR})>=0.65 \pm 0.33 \text { at }<[\mathrm{Fe} / \mathrm{H}]>=-1.58 \text {. }
$$

Although the trigonometric parallax results have large errors, these results are consistent with the statistical parallax result. All of the four results suggest the fainter absolute magnitude, $M_{V}(\mathrm{RR}) \approx 0.6-0.7$ at $[\mathrm{Fe} / \mathrm{H}]=-1.6$. Note that three estimates from parallaxes eqs.[7]-[9] are not completely independent, in regorous sense.

Recently, Feast \& Catchpole (1997) find $M_{V}(\mathrm{RR})$ $=0.25$ at $[\mathrm{Fe} / \mathrm{H}]=-1.9$, combining the LMC distance modulus of 18.7 determined by Hipparcos parallaxes of Cepheids with the data of LMC RR Lyraes by Walker (1992). On the other hand, Ried (1997) estimates the distance scale to the globular clusters by subdwarf main-sequence fitting on the bais of Hipparcos parallaxes, giving $M_{V}(\mathrm{RR})=0.15$ at $[\mathrm{Fe} / \mathrm{H}]=-$ 2.1. These bright values of $M_{V}(\mathrm{RR})$ imply much younger age for the oldest globular clusters than the previous estimates (Bolte \& Hogan 1995; Chaboyer et 
al. 1996). However, the direct estimates of $M_{V}(\mathrm{RR})$ in the present study support much fainter value than the indirect estimates obtained by them. The present results give $M_{V}(\mathrm{RR}) \approx 0.54-0.64$ at $[\mathrm{Fe} / \mathrm{H}]=-$ 1.9 , given the slope 0.20 of the $M_{V}(\mathrm{RR})-[\mathrm{Fe} / \mathrm{H}]$ relation. Note that the Walker's $M_{V}(\mathrm{RR})$ combined with the LMC distance modulus of 18.37 (Gould 1995) derived from the SN 1987A ring distance yields $M_{V}(\mathrm{RR})=0.57$ at $[\mathrm{Fe} / \mathrm{H}]=-1.9$, which favors the present results. Our findings imply that the age of the oldest globular clusters in our Galaxy still conflicts with the standard cosmological model of a flat, matterdominated universe with the Hubble constant $H_{0}=$ $60-80 \mathrm{~km} \mathrm{~s}^{-1} \mathrm{Mpc}^{-1}$ estimated from almost all observations (Bolt \& Hogan 1995). It appears that the age problem remains still unresolved.

This work has been supported in part by the grantin-Aid for Scientific Research (08640336) and Center of Excellence (COE) research (07CE2002) of the Ministry of Education, Science, and Culture in Japan. We would like to thank H. Saio for many fruitful discussions.

\section{REFERENCES}

Arenou, F., Lindegren, L., Froeschlé, M., Gómez, A. E., Turon, C., Perryman, M. A. C., \& Wielen, R. 1995, A\&A, 304, 52

Barnes III, T. G., \& Hawley, S. L. 1986, ApJ, 307, L9

Bolte, M., \& Hogan, C. J. 1995, Nature, 376, 399

Chaboyer, B., Demarque, K., \& Sarajedini, A. 1996, ApJ, 459, 558

Chaboyer, B., Demarque, K., Kernan, P. J., \& Krauss, L. M. 1996, Science, 271, 957

ESA, 1997, The Hipparcos and Tycho Catalogues, SP-1200, vol.1, chap.3

Feast, M. W., \& Catchpole, R. W. 1997, MNRAS, $286, \mathrm{~L} 1$

Fernley, J., Barnes, T.G., Skillen, I. et al. 1997, in HIPPARCOS Venice '97 Symposium, ed. M. A. C. Perryman (ESA) in press

Gould, A. 1995, ApJ, 452, 189

Hanson, R. B. 1979, MNRAS, 186, 875
Hawley, S. L., Jeffreys, W. H., \& Barnes III, T. G. 1986, ApJ, 302, 626

Jones, R. V., Carney, B. W., Storm, J., \& Latham, D. W. 1992, ApJ, 386, 646

Layden, A. C. 1994, AJ, 108, 1016

Layden, A. C., Hanson, R. B., Hawley, S. L., Klemola, A. R., Hanley, C. J. 1996, AJ, 112, 2110

Liu, T., \& Janes, K. A. 1990a, ApJ, 354, 273

Liu, T., \& Janes, K. A. 1990b, ApJ, 360, 561

Lutz, T. E., \& Kelker, D. H. 1973, PASP, 85, 573

Ratnatunga, K. U., \& Casertano, S. 1991, AJ, 101, 1075

Ried, I. N. 1997, AJ, 114, 161

Sandage, A. 1993, AJ, 106, 703

Skillen, I., Fernley, J. A., Stobie, R. S., \& Jameson, R. F. 1993, MNRAS, 265, 301

Smith, H. 1988, A\&A, 198, 365

Smith, H. 1987, A\&A, 171, 336

Storm, J., Carney, B. W., \& Latham, D. W. 1994, A\&A, 290, 443

Strugnell, P., Reid, N., \& Murray, C. A. 1986, MNRAS, 220, 413

Tsujimoto, T., Miyamoto, M., Yoshii, Y. 1997, in HIPPARCOS Venice '97 Symposium, ed. M. A. C. Perryman (ESA) in press

Walker, A. R. 1992, ApJ, 390, L81

This 2-column preprint was prepared with the AAS LATEX macros $\mathrm{v} 4.0$. 


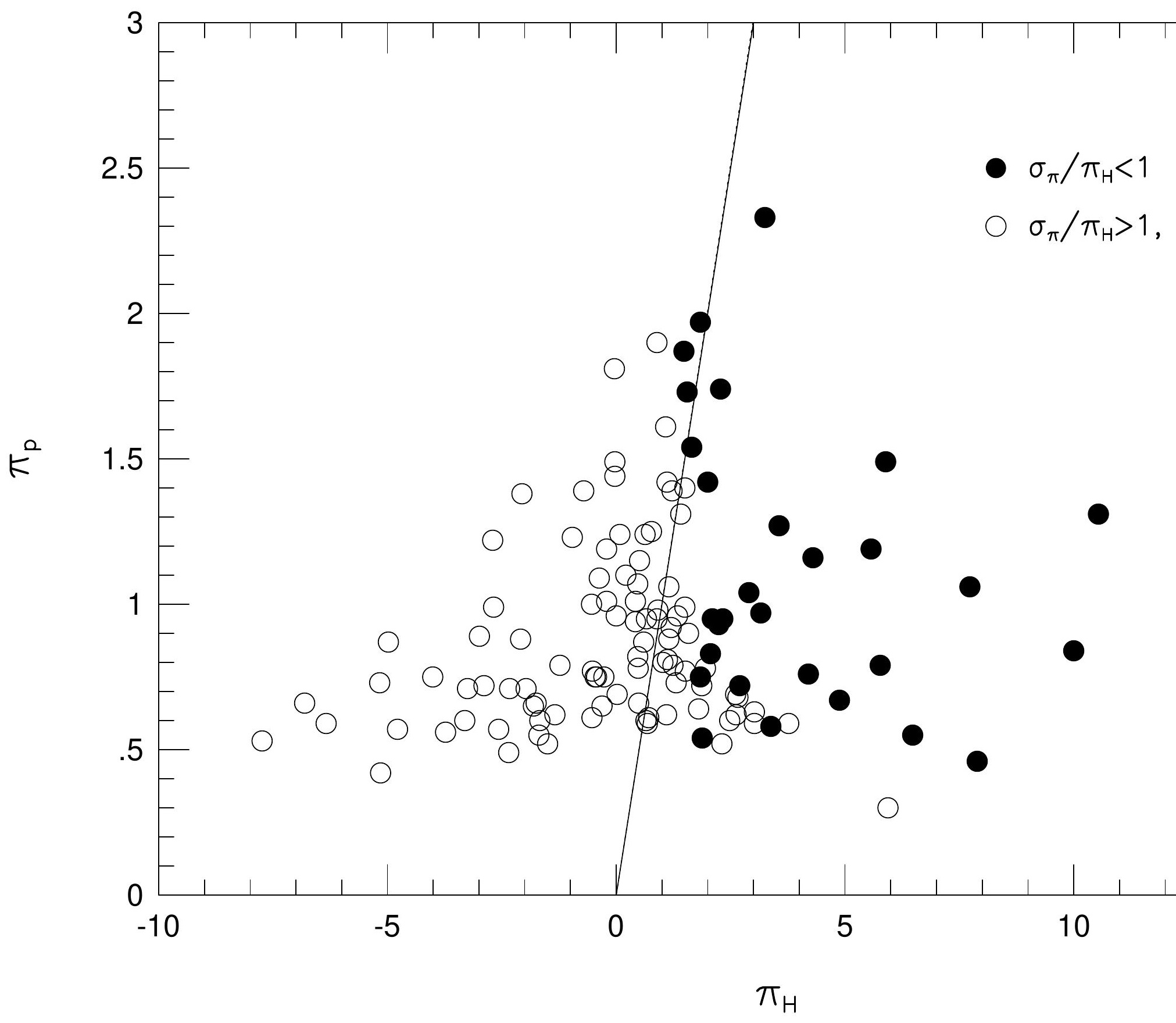

